\title{
Arithmetic Progressions Consisting Only of Primes
}

\author{
By Emil Grosswald and Peter Hagis, Jr.
}

\begin{abstract}
Let $N_{m}(x)$ denote the number of arithmetic progressions consisting of $m$ primes with largest member not exceeding $x . N_{m}(x)$ has been tabulated for $3 \leqslant m$ $\leqslant 10$ and selected values of $x$ between 1000 and 50000, and the results are compared here with those obtained by (heuristic) asymptotic approximations to $N_{m}(x)$.
\end{abstract}

1. Introduction. Although an old conjecture asserts the existence of arithmetic progressions of arbitrary length and consisting only of primes, the longest known progression of primes consists of only 17 terms and was discovered only recently [11]. In what follows, when we mention an arithmetic progression, or even simply a progression, we shall mean an arithmetic progression all of whose terms are primes. Computer searches for long arithmetic progressions have been made by Golubev ([1], [2] , [3] , [4]), Karst and Root ([9], [10]), Weintraub ([11], [12]) and others ([7] , [8]). While a fairly large number of progressions with 10 terms are known, relatively few with 11 , 12,13 or more terms have been found. In some searches negative primes were accepted (see, for example, pp. 300-301 in [3]), but we shall restrict our attention to progressions consisting of positive primes only. The letter $p$, with or without a subscript, will always denote a prime. In particular, $p_{n}$ will represent the $n$th prime so that $p_{1}=$ $2, p_{2}=3$, etc. We shall denote the common difference of any given progression by $d$.

If $p \nmid d$, then any $p$ consecutive terms of a progression constitute a complete residue system modulo $p$ so that one of the terms is divisible by $p$. It is, therefore, easy to see that for a progression with exactly $p_{n}$ terms either $d$ is divisible by $P_{n}=2 \cdot 3$ $\cdots p_{n}$ or $P_{n-1} \mid d$ and the first term of the progression is $p_{n}$. If a progression contains more than $p_{n}$ terms, then $P_{n} \mid d$. For most of the known progressions with 10 or 11 terms $d=P_{6}=2 \cdot 3 \cdot 5 \cdot 7 \cdot 11 \cdot 13=30030$. Indeed, one might well expect to find arithmetic progressions of primes with up to 16 terms having common difference $P_{6}$ but, apparently, none is known with more than 12 terms. (The smallest is $23143+$ $30030 k(k=0, \ldots, 11)$.)

A rather natural question suggests itself: If $m$ is a given positive integer, how large must $x_{m}$ be if one is to have a "reasonable" chance of finding a progression with $m$ terms, none of which exceeds $x_{m}$ ? In Table 2 we give for each $m=2,3, \ldots, 17$ the arithmetic progression with $m$ terms for which the last ( $m$ th) term $q_{m}$ is the smallest known to date. For $m \leqslant 10, q_{m}$ is the minimal value for the last term of a progression of length $m$. For $m=11,12,13$ it is highly likely that the given value of $q_{m}$ is indeed the smallest $m$ th term that exists. For $m=14,15,16,17$ this is much less

Received October 10, 1978.

AMS (MOS) subject classifications (1970). Primary 10A25, $10 \mathrm{~L} 10$. 
certain. In any event, these values of $q_{m}$ indicate rather clearly that we may expect $x_{m}$ to increase quite rapidly with $m$. One possible approach to evaluating $x_{m}$ is to derive a formula (or approximation), say $F_{m}(x)$, for $N_{m}(x)$, the number of arithmetic progressions with $m$ terms and largest member not exceeding $x$, and then determine $y_{m}$ so that $F_{m}(x) \geqslant 1$ for $x \geqslant y_{m}$. If for any given $m$ one can show that $y_{m}$ exists (and, of course, $F_{m}(x)$ is a "good" approximation to $N_{m}(x)$ ), one will have verified the existence of arithmetic progressions of arbitrary length and consisting only of primes.

Several reasonably independent methods lead to explicit asymptotic formulas for $N_{m}(x)$. In what follows we shall discuss two of these formulas which we denote by $\bar{N}_{m}(x)$ and $N_{m}^{*}(x)$, respectively. The smallest positive integer $y_{m}$ such that $\bar{N}_{m}(x) \geqslant 1$ for $x \geqslant y_{m}$ will furnish us with a (hopefully) reasonable approximation to the desired value $x_{m}$ (see Table 2). Moreover, these formulas show that for all $m, \lim _{x \rightarrow \infty} N_{m}(x)$ $=\infty$ and, hence, appear to confirm the stated conjecture. For $m \leqslant 3$, the formulas are known to be correct. For $m>3$, however, all known "proofs" make use at some point of an unproved assumption. Thus, the use of these asymptotic formulas must, at present, be considered as being only heuristically justified.

The purpose of the present paper is to compare results obtained by these asymptotic formulas with actual computer counts of $N_{m}(x)$. For practical reasons the largest value of $x$ considered was 50,000 . The results indicate that: (a) for large values of $m$ the limit 50,000 is far too low to permit any accurate prediction of the growth rate of $N_{m}(x)$; (b) the heuristic formulas are probably correct.

2. Some Heuristic Results. In 1922 Hardy and Littlewood published Part III of their famous series of "Partitio Numerorum" [6]. There, among other results, they prove a number of theorems based on plausible, but unproved, conjectures. Specifically, following Hardy and Littlewood, let $a_{1}, a_{2}, \ldots, a_{m}$ be given, distinct positive integers; and let

$$
f_{m}(x)=\sum_{p=2}^{\infty} \Lambda(p) \Lambda\left(p+a_{1}\right) \cdots \Lambda\left(p+a_{m}\right) x^{p}
$$

where $\Lambda(n)$ is von Mangoldt's function $\left(\Lambda(n)=\log p\right.$ if $n=p^{k}, \Lambda(n)=0$ otherwise). Using heuristic reasoning, one is led to Hypothesis X (see p. 56 in [6]): If $m \geqslant 0$ and $r \rightarrow 1$, then $f_{m}(r) \sim S_{m} /(1-r)$. Here $S_{m}$ is a constant, dependent on the $a_{j}$ 's, whose exact (and rather complicated) definition will not be needed in what follows.

Using Hypothesis X, Hardy and Littlewood prove six theorems. The first is the justly famous " $m$-tuples conjecture" which they call Theorem $X_{1}$. We quote this lengthy theorem in full, with some minor notational changes, in order to make the present paper self-contained.

TheOREM $\mathrm{X}_{1}$. Let $b_{1}, b_{2}, \ldots, b_{m}$ be $m$ distinct integers, and $P\left(x, b_{1}, \ldots, b_{m}\right)$ the number of groups $n+b_{1}, n+b_{2}, \ldots, n+b_{m}$ between 1 and $x$ and consisting wholly of primes. Then

$$
P(x) \sim G\left(b_{1}, b_{2}, \ldots, b_{m}\right) \cdot L i_{m}(x) \quad \text { when } x \rightarrow \infty
$$


where

$$
G\left(b_{1}, \ldots, b_{m}\right)=\prod_{p \geqslant 2}\left(\frac{p}{p-1}\right)^{m-1}\left(\frac{p-\nu}{p-1}\right)
$$

$\nu=\nu\left(p, b_{1}, \ldots, b_{m}\right)$ is the number of distinct residues modulo $p$ that occur in the set $\left\{b_{1}, b_{2}, \ldots, b_{m}\right\}$, and

$$
L i_{m}(x)=\int_{2}^{x} \frac{d \mu}{(\log \mu)^{m}}
$$

Further,

$$
G\left(b_{1}, b_{2}, \ldots, b_{m}\right)=D_{m} \cdot H\left(b_{1}, b_{2}, \ldots, b_{m}\right)
$$

where

$$
\begin{gathered}
D_{m}=\prod_{p>m}\left\{\left(\frac{p}{p-1}\right)^{m-1}\left(\frac{p-m}{p-1}\right)\right\}, \\
H\left(b_{1}, b_{2}, \ldots, b_{m}\right)=\prod_{p \leqslant m}\left\{\left(\frac{p}{p-1}\right)^{m-1}\left(\frac{p-v}{p-1}\right)\right\} \prod_{\substack{p \mid \Delta \\
p>m}}\left(\frac{p-v}{p-m}\right),
\end{gathered}
$$

and $\Delta$ is the product of the differences of the $b_{j}$ 's.

On the basis of Theorem $X_{1}$ one of the present authors has proved (see [5]) the following result.

THEOREM. Let $m \geqslant 2$, and denote by $N_{m}(x)$ the number of arithmetic progressions of $m$ terms consisting only of primes, none larger than $x$. Define the constant

$$
C_{m}=\prod_{p \leqslant m}\left\{\frac{1}{p}\left(\frac{p}{p-1}\right)^{m-1}\right\} \prod_{p>m}\left\{\left(\frac{p}{p-1}\right)^{m-1}\left(\frac{p-m+1}{p}\right)\right\}
$$

and set

$$
\bar{N}_{m}(x)=\frac{C_{m}}{2(m-1)} \cdot \frac{x^{2}}{\log ^{m} x} .
$$

Then (assuming Theorem $\mathrm{X}_{1}$ )

$$
N_{m}(x) \sim \bar{N}_{m}(x)
$$

The factor $1 / 2(m-1) \cdot x^{2} / \log ^{m} x$ in $(2)$ is the asymptotic value of the sum

$$
S_{m}(x)=\sum\left(\log n_{1} \cdot \log n_{2}, \ldots, \log n_{m}\right)^{-1} .
$$

Here the summation is extended over all $m$-tuples $n_{1}, n_{2}, \ldots, n_{m}$ of positive integers such that $n_{1}<n_{2}<\cdots<n_{m}$ are in arithmetic progression and $2 \leqslant n_{1}, n_{m} \leqslant x$. Indeed, making use of a highly nontrivial application of the Euler-Maclaurin formula it is shown in [5] that $S_{m}(x)$ can be represented by an asymptotic series as follows:

$$
S_{m}(x)=\frac{x^{2}}{2(m-1) \log ^{m} x}\left\{1+\sum_{j=1}^{N} \frac{a_{j}(m)}{\log ^{j} x}+O\left(\frac{1}{\log ^{N+1} x}\right)\right\},
$$

where all the $a_{j}(m)$ are computable and $N$ may be taken arbitrarily large. The series itself in (5) does not converge. 
At least two other methods lead, but still only heuristically, to (3). One, due to D. Zagier [13], is based on the assumption of the independence of the distribution of residue classes modulo distinct primes (regardless of the length of the intervals considered). The other is based on Vinogradov's version of the "circle method" of Hardy, Littlewood and Ramanujan. For $m=3$, (3) (and, in fact, somewhat more) has been obtained by this approach without the use of any unproved hypotheses (see [5]); but for $m \geqslant 4$ the technical difficulties could not be overcome. However, if one proceeds formally, ignoring such difficulties as large error terms, etc., one obtains $N_{m}(x)$ as the product of a "singular series" and the sum in (4).

In view of the fact that several methods lead to (3), but that none of them (thus far) provide a convincing proof if $m \geqslant 4$, it appears desirable at this time to investigate to what extent actual calculations are in agreement with (3).

3. Auxiliary Considerations. Before we describe the results obtained a few remarks are in order. From (2) and (5), for $m \geqslant 2,(3)$ is equivalent to

$$
N_{m}(x) \sim N_{m}^{*}(x)=C_{m} S_{m}(x),
$$

where $S_{m}(x)$ is given by (4). However, (6), is meaningful also for $m=1$ which is not the case for (3). In fact, (6) is true both for $m=1$ and $m=2$ as we easily verify as follows.

If $m=1$ then, from (1), $C_{1}=1$ and $N_{1}^{*}(x)=\Sigma_{n=2}^{x}(1 / \log n)=\operatorname{li} x+O(1)$, where li $x$ is the integral logarithm. Since every prime is an arithmetic progression of length one, $N_{1}(x)=\pi(x)$; and since, by the Prime Number Theorem, $\pi(x) \sim \operatorname{li} x$, it follows that $N_{1}(x) \sim N_{1}^{*}(x)$.

More can be said. For, by the Prime Number Theorem, $\pi(x)=\operatorname{li} x+O\left(x e^{-c \sqrt{\log x}}\right)$ $=$ li $x+O\left(x \log ^{-M} x\right)$, where $M$ is any positive integer. Therefore, from the classical asymptotic series for li $x$, we have

$$
\begin{aligned}
& N_{1}(x)=\pi(x)=\frac{x}{\log x}\left\{1+\frac{1 !}{\log x}+\frac{2 !}{\log ^{2} x}+\cdots+\frac{N !}{\log ^{N} x}+O\left(\frac{1}{\log ^{N+1} x}\right)\right\} \\
& \begin{aligned}
\text { If } m=2, \text { then } C_{2}=1 \text { and } \\
N_{2}^{*}(x)=\sum_{\substack{n_{2}=3 \\
2 \leqslant n_{1}<n_{2}}}^{x}\left(\log n_{1} \cdot \log n_{2}\right)^{-1}=1 / 2\left\{\left(\sum_{n=2}^{x} \frac{1}{\log n}\right)^{2}-\sum_{n=2}^{x} \log ^{-2} n\right\} \\
=1 / 2\left\{(\operatorname{li} x+O(1))^{2}+O(\operatorname{li} x)\right\}=1 / 2 \operatorname{li}^{2} x+O(\operatorname{li} x) \\
=1 / 2 \operatorname{li}^{2} x(1+O(\log x / x)),
\end{aligned}
\end{aligned}
$$

where we have used the fact that li $x \sim x / \log x$. On the other hand, since every pair of distinct primes is an arithmetic progression of length two, it is clear that $N_{2}(x)$ is the number of combinations of $\pi(x)$ primes taken two at a time. Therefore,

$$
\begin{aligned}
N_{2}(x) & =1 / 2 \pi(x)[\pi(x)-1]=1 / 2\left\{\operatorname{li} x+O\left(x e^{-c \sqrt{\log x}}\right)\right\}\left\{\operatorname{li} x+O\left(x e^{-c \sqrt{\log x}}\right)\right\} \\
& =1 / 2 \operatorname{li}^{2} x(1+o(1)) ;
\end{aligned}
$$

and it follows that $N_{2}(x) \sim N_{2}^{*}(x)$. Again using the asymptotic series for li $x$, we 
obtain

$$
\begin{aligned}
N_{2}(x) & =1 / 2 \pi(x)[\pi(x)-1]=1 / 2\left[\operatorname{li} x+O\left(x \log ^{-M-1} x\right)\right]\left[\operatorname{li} x+O\left(x \log ^{-M-1} x\right)\right] \\
& =1 / 2 \operatorname{li}^{2} x\left[1+O\left(\frac{1}{\log ^{M} x}\right)\right] \\
& =\frac{x^{2}}{2 \log ^{2} x}\left\{1+\frac{2}{\log x}+\frac{5}{\log ^{2} x}+\cdots+\frac{a_{N}(2)}{\log ^{N} x}+O\left(\frac{1}{\log ^{N+1} x}\right)\right\}
\end{aligned}
$$

We now call attention to the following rather unpleasant computational fact. For a relatively small value of $x$, say $10^{4}, \log x \approx 9.2,2 / \log x>.21$ and $5 / \log ^{2} x>.05$. Hence, for $m=2$ and $x=10^{4},(5)$ is affected by an error in excess of $26 \%$ if we neglect the "corrective terms" in the asymptotic formula for $S_{m}(x)$. The situation is much worse for larger values of $m$.

If $m=3$, (1) yields $C_{3}=2 \Pi_{p \neq 2}\left(1-(p-1)^{-2}\right)=2 C_{0}$, where $C_{0}=.66016 \ldots$, the "twin primes" constant. From (2) and (3), $N_{3}(x) \sim\left(C_{0} / 2\right) x^{2} / \log ^{3} x$. In fact, it is known (see [5]) that

$$
N_{3}(x)=\frac{C_{0}}{2} \cdot \frac{x^{2}}{\log ^{3} x}\left\{1+\frac{a_{1}}{\log x}+\frac{a_{2}}{\log ^{2} x}+O\left(\frac{1}{\log ^{3} x}\right)\right\},
$$

where $a_{1}=3.5-\log 2>2.8$ and $a_{2}=13-5 \log 2-\log ^{2} 2-\pi^{2} / 12 \approx 8.231344049 \ldots$ If one so desires, any number of coefficients of the asymptotic series represented by the bracket in the expression for $N_{3}(x)$ just given can be computed. If $x=10^{4}$, then $a_{1} / \log x>3$. If we neglect this term, our error exceeds $30 \%$. As in the case $m=2$ this example shows that if we want to compare in some meaningful way actual values of $N_{m}(x)$ with values obtained by our formulas, we have to choose among the following options:

(i) formulas (2) and (3) may be used if large enough values of $x$ are utilized so that the neglected terms of the asymptotic series are indeed negligible;

(ii) formula (6) may be used;

(iii) the first terms of the asymptotic expansion in (5) may be used so that (6) becomes

$$
N_{m}(x) \sim \frac{C_{m}}{2(m-1)} \cdot \frac{x}{\log ^{m} x}\left\{1+\frac{a_{1}(m)}{\log x}+\cdots+\frac{a_{N}(m)}{\log ^{N} x}+O\left(\frac{1}{\log ^{N+1} x}\right)\right\} .
$$

The need for these corrective terms, at moderate values of $x$, was already recognized by Hardy and Littlewood (see pp. 37-38 in [6]).

The values of $x$ required by (i) are beyond the practical range of present day computers for counting $N_{m}(x)$. On the other hand, the computation of the coefficients $a_{j}(m)$ required by (iii), quite easy if $m$ is small, becomes very difficult for large values of $m$. These considerations led us to rely primarily on (ii) in the present paper.

4. A Description of Our Tables and Some Comments. Using the CDC 6400 at the Temple University Computer Center, the values of $N_{m}(x), \bar{N}_{m}(x)$ (see (2)), and $N_{m}^{*}(x)$ (see (4) and (6)) were calculated for $m=3(1) 10, x=1000(1000) 10000$ and 
$x=10000(10000) 50000$ and for $m=3,4,5$ are given in Table. 1. (An extension of Table 1 which covers the cases $m=6$ to $m=10$ is available from the authors upon request.) The required values of $C_{m}$ were computed by truncating the infinite product in (1) at $p=999983$, and estimating the error thus incurred. The results (correct to six significant digits for $m<15$ and five significant digits for $15 \leqslant m \leqslant 20$ ) appear in Table 3. The values of $S_{m}(x)$ were obtained by a rather straightforward application of definition (4). Thirteen significant digits were carried in the calculations, and the results were rounded to seven digits to minimize roundoff error.

The ratios $N_{m}(x) / N_{m}^{*}(x), N_{m}(x) / \bar{N}_{m}(x)$ and $N_{m}^{*}(x) / \bar{N}_{m}(x)$ are also given in Table 1. If the conjectural (for $m \geqslant 4$ ) formulas are correct these ratios will all approach unity as $x \rightarrow \infty$. Otherwise, only $N_{m}^{*}(x) / \bar{N}_{m}(x)$ may be expected to converge to one.

Since (3) and (6) are known to be true for $m=3$ but (as of this writing) are still only conjectural for $m \geqslant 4$, the speed of convergence of $N_{m}(x) / N_{m}^{*}(x)$ and $N_{m}(x) / \bar{N}_{m}(x)$ to unity in the former case may be indicative of what could be expected in the cases $m \geqslant 4$ if (3) and (6) are indeed true for all $m$. In this context we observe that the ratio $N_{3}(x) / N_{3}^{*}(x)$ (the more significant of the two) increases from .841 for $x=1000$ to $.956 \ldots$ for $x=10000$ to $.980 \ldots$ for $x=50000$. For $m=4$ the corresponding values increase from $.693 \ldots$ to $.927 \ldots$ to $.970 \ldots$ However, already for $m=6$ the figures deteriorate to $.371 \ldots, .695 \ldots, .895 \ldots$, respectively. For $m=7$ the figures are $.079 \ldots, .439 \ldots, .789 \ldots$; for $m=8$ they are $0, .270 \ldots, .680 \ldots$; for $m=9$ they are $0, .221 \ldots, .464 \ldots$ An eventual convergence to unity is still suggested, but the rate of convergence appears to diminish rapidly as $m$ increases.

TABLE 1

\begin{tabular}{cllllll}
\hline$x$ & $\mathrm{~N}_{3}(\mathrm{x})$ & $\mathrm{N}_{3}^{*}(\mathrm{x})$ & $\overline{\mathrm{N}}_{3}(\mathrm{x})$ & $\mathrm{N}_{3} / \mathrm{N}_{3}^{*}$ & $\mathrm{~N}_{3} / \overline{\mathrm{N}}_{3}$ & $\mathrm{~N}_{3}^{*} / \overline{\mathrm{N}}_{3}$ \\
\hline 1000 & 1500 & 1782.4 & 1001.4 & .84155 & 1.4979 & 1.7799 \\
2000 & 4457 & 4980.5 & 3006.7 & .89489 & 1.4824 & 1.6565 \\
3000 & 8478 & 9255.5 & 5788.4 & .91600 & 1.4647 & 1.5990 \\
4000 & 13356 & 14472 & 9256.4 & .92290 & 1.4429 & 1.5634 \\
5000 & 19174 & 20548 & 13356 & .93315 & 1.4356 & 1.5385 \\
6000 & 25679 & 27426 & 18048 & .93630 & 1.4228 & 1.5196 \\
7000 & 33319 & 35064 & 23305 & .95024 & 1.4297 & 1.5046 \\
8000 & 41029 & 43427 & 29102 & .94478 & 1.4098 & 1.4922 \\
9000 & 49721 & 52488 & 35422 & .94728 & 1.4037 & 1.4818 \\
10000 & 59504 & 62224 & 42247 & .95629 & 1.4085 & 1.4729 \\
20000 & 186647 & 193195 & 135930 & .96611 & 1.3731 & 1.4213 \\
30000 & 368304 & 378543 & 271156 & .97295 & 1.3583 & 1.3690 \\
40000 & 599294 & 612470 & 443850 & .97849 & 1.3502 & 1.3799 \\
50000 & 873953 & 891421 & 651487 & .98040 & 1.3415 & 1.3683
\end{tabular}




\begin{tabular}{cllllll}
\hline$x$ & $\mathrm{~N}_{4}(\mathrm{x})$ & $\mathrm{N}_{4}^{*}(\mathrm{x})$ & $\overline{\mathrm{N}}_{4}(\mathrm{x})$ & $\mathrm{N}_{4} / \mathrm{N}_{4}^{*}$ & $\mathrm{~N}_{4} / \overline{\mathrm{N}}_{4}$ & $\mathrm{~N}_{4}^{*} / \overline{\mathrm{N}}_{4}$ \\
\hline 1000 & 318 & 458.36 & 209.22 & .69378 & 1.5199 & 2.1908 \\
2000 & 914 & 1126.1 & 570.88 & .81162 & 1.6010 & 1.9726 \\
3000 & 1670 & 1956.4 & 1043.4 & .85361 & 1.6005 & 1.8750 \\
4000 & 2555 & 2925.1 & 1610.7 & .87347 & 1.5863 & 1.8161 \\
5000 & 3568 & 4017.8 & 2263.1 & .88805 & 1.5766 & 1.7754 \\
6000 & 4704 & 5224.3 & 2994.1 & .90040 & 1.5711 & 1.7449 \\
7000 & 6004 & 6537.3 & 3798.9 & .91843 & 1.5805 & 1.7208 \\
8000 & 7304 & 7950.6 & 4673.4 & .91867 & 1.5629 & 1.7013 \\
9000 & 8682 & 9459.6 & 5614.6 & .91780 & 1.5463 & 1.6848 \\
10000 & 10257 & 11060 & 6619.8 & .92738 & 1.5494 & 1.6708 \\
20000 & 29811 & 31516 & 19809 & .94589 & 1.5049 & 1.5910 \\
30000 & 56528 & 58943 & 37961 & .95902 & 1.4891 & 1.5528 \\
40000 & 89345 & 92400 & 60450 & .96693 & 1.4780 & 1.5285 \\
50000 & 127397 & 131323 & 86899 & .97010 & 1.4660 & 1.5112
\end{tabular}

$m=5$

\begin{tabular}{|c|c|c|c|c|c|c|}
\hline$x$ & $N_{5}(x)$ & $N_{5}^{\star}(x)$ & $\bar{N}_{5}(x)$ & $\mathrm{N}_{5} / \mathrm{N}_{5}^{\star}$ & $\mathrm{N}_{5} / \bar{N}_{5}$ & $\mathrm{~N}_{5} / \overline{\mathrm{N}}_{5}$ \\
\hline 1000 & 58 & 93.881 & 32.991 & .61780 & 1.7580 & 2.8456 \\
\hline 2000 & 141 & 199.61 & 81.812 & .70639 & 1.7235 & 2.4398 \\
\hline 3000 & 245 & 321.74 & 141.95 & .76148 & 1.7259 & 2.2665 \\
\hline 4000 & 352 & 457.95 & 211.53 & .76864 & 1.6641 & 2.1650 \\
\hline 5000 & 464 & 606.69 & 289.43 & .76480 & 1.6032 & 2.0962 \\
\hline 6000 & 604 & 766.88 & 374.89 & .78761 & 1.6111 & 2.0456 \\
\hline 7000 & 780 & 937.67 & 467.37 & .83185 & 1.6689 & 2.0063 \\
\hline 8000 & 923 & 1118.4 & 566.42 & .82529 & 1.6295 & 1.9745 \\
\hline 9000 & 1091 & 1308.5 & 671.69 & .83376 & 1.6243 & 1.9481 \\
\hline 10000 & 1283 & 1507.6 & 782.89 & .85100 & 1.6388 & 1.9257 \\
\hline 20000 & 3484 & 3925.5 & 2178.7 & .88752 & 1.5991 & 1.8018 \\
\hline 30000 & 6383 & 6995.4 & 4011.0 & .91246 & 1.5914 & 1.7441 \\
\hline 40000 & 9894 & 10614 & 6213.9 & .93216 & 1.5922 & 1.7081 \\
\hline 50000 & 13835 & 14721 & 8748.4 & .93982 & 1.5814 & 1.6827 \\
\hline
\end{tabular}

For "large" values of $m$ the upper bound $x=50000$ of our computations is totally inadequate for drawing meaningful quantitative conclusions. This is clearly illustrated by our findings for the case $m=10 . N_{10}(x) / N_{10}^{*}(x)$ varies from 0 for $x=1000$ to $.143 \ldots$ for $x=10000$ and then decreases to $.082 \ldots$ for $x=50000$. This erratic 
behavior is very simply explained. The first progression of 10 terms is $199+210 k$ $(k=0, \ldots, 9)$; the next two are $34913+2100 k$ and $52879+420 k(k=0, \ldots, 9)$ with last terms 53813 and 56659 , respectively. Consequently, the ratio $N_{10}(x) / N_{10}^{*}(x)$ almost triples from about .08 for $x=50000$ to about .23 for $x=57000$.

Table 2 has already been described in Section 1 . For $5 \leqslant m \leqslant 20, y_{m}$ correct to four significant digits, is the smallest integer such that $\bar{N}_{m}(x)>1$ for $x \geqslant y_{m}$. The value of $y_{m}$ is not tabulated for $m=2,3,4$ since $\bar{N}_{m}(x)>1$ for these values of $m$ if $x \geqslant 2$.

TABLE 2

\begin{tabular}{|c|c|c|c|}
\hline m & Progression with minimal last term & $q_{m}$ & $y_{m}$ \\
\hline 2 & 2,3 & 3 & - \\
\hline 3 & $3,5,7$ & 7 & - \\
\hline 4 & $5,11,17,23$ & 23 & - \\
\hline 5 & $5,11,17,23,29$ & 29 & 29 \\
\hline 6 & $7+30 k *$ & 157 & 92 \\
\hline 7 & $7+150 k$ & 907 & 497 \\
\hline 8 & $199+210 k$ & 1669 & 1406 \\
\hline 9 & $199+210 k$ & 1879 & 5086 \\
\hline 10 & $199+210 k$ & 2089 & 24310 \\
\hline 11 & $110437+13860 k$ & 249037 & 177300 \\
\hline 12 & $110437+13860 k$ & 262897 & 829800 \\
\hline 13 & $4943+60060 k$ & 725663 & 5582000 \\
\hline 14 & $46883579+2462460 k$ & 78895559 & $2.332 \times 10^{7}$ \\
\hline 15 & $53297929+9699690 k$ & 189093589 & $1.137 \times 10^{8}$ \\
\hline 16 & $53297929+9699690 k$ & 198793279 & $6.793 \times 10^{8}$ \\
\hline 17 & $3430751869+87297210 k$ & 4827507229 & $5.774 \times 10^{9}$ \\
\hline 18 & - & - & $3.303 \times 10^{10}$ \\
\hline 19 & - & - & $2.564 \times 10^{11}$ \\
\hline 20 & - & - & $1.261 \times 10^{12}$ \\
\hline
\end{tabular}

In each case $k=0,1, \ldots, m-1$.

The fact that $y_{5}=q_{5}$ is, of course, coincidental. In fact, one cannot expect $y_{m}$ to approximate $q_{m}$ too closely. Indeed, this is already precluded by the fact that $\bar{N}_{m}(x)$ has been used to find $y_{m}$ rather than $N_{m}^{*}(x)$ and all the corrective terms of the asymptotic series (5) have been neglected. Furthermore, for small values of $x$ the arithmetic progressions of length $m$ with largest term not exceeding $x$ are distributed in a very irregular manner as we saw in the case $m=10$. Under these circumstances it is rather remarkable how well $y_{m}$ seems to indicate the correct order of magnitude of $q_{m}$. Table 2 also "explains" why it is necessary to go to progressions with such large terms 
in order to find arithmetic progressions with a modest length like $m=16$ or 17 . Finally, Table 2 indicates that if one is seeking an arithmetic progression of, say, 20 terms one must expect its last term to have about 13 digits.

TABLE 3

\begin{tabular}{|r|r|}
\hline$m$ & $C_{m}$ \\
\hline 3 & 1.32032 \\
4 & 2.85825 \\
5 & 4.15118 \\
6 & 10.1318 \\
7 & 17.2986 \\
8 & 53.9720 \\
9 & 148.552 \\
10 & 336.034 \\
11 & 511.422 \\
12 & 1312.32 \\
13 & 2364.60 \\
14 & 7820.61 \\
15 & 22939 \\
16 & 55651 \\
17 & 91555 \\
18 & 256480 \\
19 & 510990 \\
20 & 1901000 \\
\hline
\end{tabular}

5. Final Conclusions. While the upper bound, $x=50000$, of our computations is far too small to permit any quantitative inferences, especially for $m>6$, the validity of the heuristic formulas (3) and (6) is strongly suggested by our computer data. This conclusion is reinforced when one compares the rates of convergence of the ratios $N_{m}(x) / N_{m}^{*}(x)$ and $N_{m}(x) / \bar{N}_{m}(x)$ to one in the case $m=3$ (when (3) and (6) are known to be valid) with those in the cases $m=4$ and $m=5$ and when one observes the (overall) agreement of the orders of magnitude of $q_{m}$ and $y_{m}$.

Department of Mathematics

Temple University

Philadelphia, Pennsylvania 19122

1. W. A. GOLUBEV, "Faktorisation der Zahlen der Form $x^{3} \pm 4 x^{2}+3 x \pm 1$," Anz. Oesterreich. Akad. Wiss. Math.-Naturwiss. Kl., 1969, pp. 184-191.

2. W. A. GOLUBEV, "Faktorisation der Zahlen der Form $x^{3} \pm 57$," Anz. Oesterreich. Akad. Wiss. Math.-Naturwiss. Kl., 1969, pp. 191-194.

3. W. A. GOLUBEV, "Faktorisation der Zahlen der Formen $x^{3} \pm 83$ und $x^{3} \pm 92009$," Anz. Oesterreich. Akad. Wiss. Math.-Naturwiss. Kl., 1969, pp. 297-301. 
4. W. 'A. GOLUBEV, "Faktorisation der Zahlen der Form $x^{3}+4 x^{2}-25 x+13$," Anz. Oesterreich. Akad. Wiss. Math.-Naturwiss. Kl., 1970, pp. 106-112.

5. E. GROSSWALD, "Arithmetic progressions of primes." (To appear.)

6. G. H. HARDY \& J. E. LITTLEWOOD, "Some problems of 'Partitio Numerorum'; III: On the expression of a number as a sum of primes," Acta Math., v. 44, 1922, pp. 1-70.

7. E. KARST, " 12 to 16 primes in arithmetical progression," J. Recreational Math., v. 2, 1969, pp. 214-215.

8. E. KARST, "Lists of ten or more primes in arithmetical progression," Scripta Math., v. 28, 1970, pp. 313-317.

9. E. KARST \& S. C. ROOT, "Teilfolgen von Primzahlen in arithmetischer Progression," Anz. Oesterreich. Akad. Wiss. Math.-Naturwiss. Kl., 1972, pp. 19-20.

10. S. C. ROOT \& E. KARST, "Mehr Teilfolgen von Primzahlen in arithmetischer Progression," Anz. Oesterreich. Akad. Wiss. Math.-Naturwiss. Kl., 1972, pp. 178-179.

11. S. WEINTRAUB, "Seventeen primes in arithmetic progression," Math. Comp., v. 31, 1977 , p. 1030 .

12. S. WEINTRAUB, "Primes in arithmetic progression," $B I T$, v. 17, 1977, pp. 239-243.

13. D. ZAGIER, Private communication. 